DESY 96-060

ISSN 0418-9833

April 1996

\title{
Unified Description of Rapidity Gaps and Energy Flows in DIS Final States
}

\author{
A. Edin ${ }^{1}$, G. Ingelman ${ }^{1,2}$, J. Rathsman ${ }^{1}$ \\ ${ }^{1}$ Dept. of Radiation Sciences, Uppsala University, Box 535, S-751 21 Uppsala, Sweden \\ ${ }^{2}$ Deutsches Elektronen-Synchrotron DESY, Notkestrasse 85, D-22603 Hamburg, Germany
}

\begin{abstract}
We show that the 'orthogonal' characteristics of the observed rapidity gaps and large forward energy flows in deep inelastic scattering at HERA, can be described within a single framework. Our Monte Carlo model is based on perturbative QCD matrix elements and parton showers together with Lund string model hadronization, but has in addition a new mechanism for soft colour interactions which modifies the perturbative colour structure and thereby the hadronization. Effects of perturbative multiparton emission are investigated and the non-perturbative treatment of the proton remnant is discussed and comparison to the observed transverse energy flow is made. We investigate the resulting diffractive-like properties of the model; such as rapidity gap events, $t$ - and $M_{X}$-distributions and the diffractive structure function in comparison to $\mathrm{H} 1$ data.
\end{abstract}

\section{Introduction}

The hadronic final state in deep inelastic scattering (DIS) of leptons on nucleons provides information on strong interaction dynamics. The availability of many different observables, such as particle and energy flows, jet structures etc., give possibilities for investigations of perturbative and non-perturbative QCD in extension to those based on measurements of inclusive structure functions.

Valuable results from final state investigations have been obtained at various fixed target experiments and with the electron-proton collider HERA at DESY a large new kinematic domain has been made available. In particular, the much larger range in mass $W$ of the hadronic system, extending up to the kinematic limit $\sim 300 \mathrm{GeV}$, provides an increased phase space for parton emission and make multi-jet events possible. Connected to large $W$ is also the extension of Bjorken- $x$ down to $\sim 10^{-4}$ in DIS. New initial state radiation effects can then appear, such as BFKL-dynamics [1] where resummation of large $\log (1 / x)$ terms may become noticeable in the structure function or the characteristic nonordering in transverse momenta of emitted gluons observable in forward energy flows or jets.

Two main new observations have already been made in the small- $x$ data collected by H1 and ZEUS collaborations at HERA. The first is the large mean transverse energy at forward rapidities [2, 3] (i.e. in the proton beam hemisphere), which may be a signal for 
BFKL dynamics. In contrast to this mean behaviour, the second effect is the discovery [4, 5] that about $10 \%$ of the events have no particles or energy depositions in the forward part of the detector, i.e. the final state has a large gap in rapidity space. These two features of the data are thus 'orthogonal' and hard to account for in one and the same theoretical framework. The description of the energy flows requires a sufficient amount of perturbative parton emission and non-perturbative effects from hadronization. The gap events, on the other hand, require the absence of parton and hadron emissions in the rapidity region of the gap. A main question is then whether these gaps can occur as fluctuations in perturbative QCD and hadronization, with some new dynamical assumptions, or whether their understanding requires the addition of a conceptually different mechanism such as diffractive interactions based on pomeron exchange where there is no continuous transition between the two phenomena.

Given the complexity of the final states and these observables, a detailed comparison with theoretical models is best performed using Monte Carlo simulation methods. Standard programs, like LEPTO [6] and ARIADNE [7], for DIS have been totally unable to describe the rapidity gap events. The gap events can, on the other hand, be reasonably well described in terms of diffractive interactions as given by pomeron exchange in Regge phenomenology. The basic idea is that the pomeron has a parton content which can be probed in hard scattering processes [8]. Monte Carlo models, such as POMPYт [9] and RAPGAP [10], based on these notions can give a reasonable description of the data. In spite of this, there is no satisfactory understanding of the pomeron and its interaction mechanisms. It is therefore of interest to consider alternative ways to understand the rapidity gap phenomenon.

We have previously introduced [11] a novel soft colour interaction (SCI) mechanism to understand the large rapidity gap events in a new way, without any explicit use of the pomeron concept. Our approach is similar in spirit to the one in ref. [12]. Their model considers only the first order QCD matrix element, where the whole DIS cross-section is being saturated by the boson-gluon fusion process, and introduces a statistical probability that the produced $q \bar{q}$ pair is changed from a colour octet into a colour singlet state. In our model, the influence of higher order parton emissions are included, an explicit mechanism for the colour exchange is introduced and hadronization is taken into account. Having formulated our model in terms of a Monte Carlo generator that simulates the complete final state, more detailed comparisons with data can be made. Adding our SCI model to the normal Monte Carlo program LEPTO [13] for DIS, we describe the salient features of the HERA data, both regarding rapidity gaps and energy flows.

In this paper we make a more comprehensive discussion of our model and describe its parts in more detail. The perturbative QCD aspects, i.e. matrix elements and parton showers, are discussed in section 2. The non-perturbative part of the model, i.e. soft colour interactions, target remnant treatment and hadronization, are described in section 3. In section 4 , we show the most relevant results in terms of observable quantities and make some comparison with data. Finally, we give a concluding discussion in section 5 .

\section{Perturbative QCD effects}

The starting point for describing the hadronic final state is the deep inelastic scattering on a quark in the proton as given by standard electroweak cross sections for $\gamma, Z, W$ exchange [13]. Perturbative QCD (pQCD) effects are then taken into account through 
leading order matrix elements and higher orders through parton shower approximations. These two parts are discussed in the following, together with their interrelation.

\subsection{Matrix elements}

Hard parton processes are best described by exact matrix elements, but one is then limited to low orders in the perturbative expansion. The two leading order QCD processes QCD Compton (QCD-C) and boson-gluon fusion (BGF) are shown in Fig. 1, which also defines the relevant four-momenta. The two processes result in a quark-gluon and a quarkantiquark parton system, respectively, and are labeled by $q g$ and $q \bar{q}$. The cross-section is calculated by folding the proton's parton density functions (for which we use CTEQ2L 14) with the hard scattering cross-sections $d \hat{\sigma}_{q g}$ and $d \hat{\sigma}_{q \bar{q}}$ [15, 16]

$$
\begin{aligned}
d \sigma_{M E}\left(x, Q^{2}, x_{p}, z_{q}, \phi\right) \propto & \sum_{f} q_{f}\left(x / x_{p}, Q^{2}\right) \otimes d \hat{\sigma}_{q g}\left(x, Q^{2}, x_{p}, z_{q}, \phi\right) \\
& +g\left(x / x_{p}, Q^{2}\right) \otimes d \hat{\sigma}_{q \bar{q}}\left(x, Q^{2}, x_{p}, z_{q}, \phi\right) .
\end{aligned}
$$

$Q^{2}=-\left(p_{l}-p_{l^{\prime}}\right)^{2}$ and $x=Q^{2} / 2 P \cdot q$ are the usual deep inelastic variables that are sufficient to describe the inclusive lepton scattering. The three additional degrees of freedom in the first order processes are usually described in terms of $x_{p}=x / \xi, z_{q}=P \cdot j_{1} / P \cdot q$ and the azimuthal angle $\phi$ between the lepton scattering plane and the parton scattering plane. The incoming parton momentum $k=\xi P$ is a fraction $\xi$ of the proton's momentum. The two produced partons have invariant mass squared $\hat{s}=\left(j_{1}+j_{2}\right)^{2}$, whereas the whole hadronic system has invariant mass squared $W^{2}=(P+q)^{2}$.
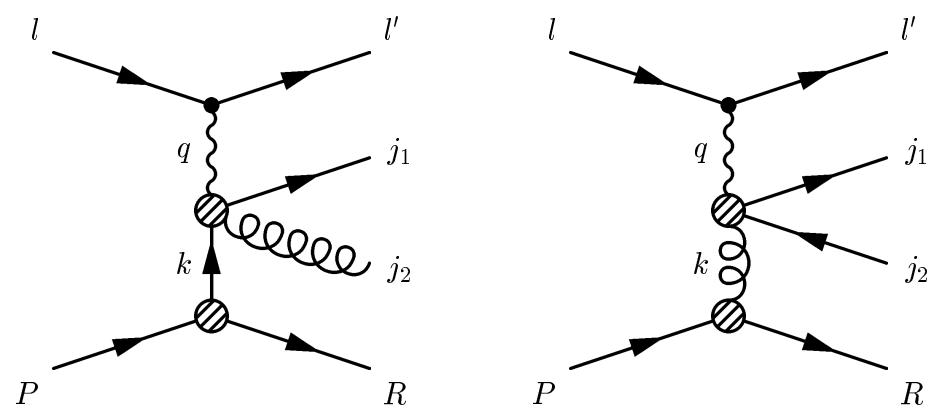

Figure 1: First order QCD processes in DIS: (a) QCD Compton $\gamma^{*} q \rightarrow q g$, (b) bosongluon fusion $(\mathrm{BGF}) \gamma^{*} g \rightarrow q \bar{q}$.

These matrix elements are often discussed only in connection with cross sections for observable jets, corresponding to hard and well separated partons. Here we want to describe the hadronic system more generally and therefore also make extensions into the region where the hard subsystem has a small invariant mass squared $\hat{s}$. One then has to care about the divergences in the matrix elements, which for the QCD-Compton part $\hat{\sigma}_{q g}$ behaves as $1 /\left(1-x_{p}\right)\left(1-z_{q}\right)$ and for the boson gluon fusion part $\hat{\sigma}_{q \bar{q}}$ as $1 / z_{q}\left(1-z_{q}\right)$ [15, 16]. In a Monte Carlo simulation, these are usually avoided by a simple cut-off, although a more elaborate procedure based on Sudakov form factors is also possible [17]. There exist several different cut-off schemes which have been considered [16]. Most commonly used has been the $W$-scheme with the requirement $s_{i j}=\left(p_{i}+p_{j}\right)^{2}>y_{\text {cut }} W^{2}$ for any pair $i j$ of partons. Here, not only $j_{1}, j_{2}$ but also the remnant parton $R=(1-\xi) p$ is included to 
provide a cut against soft and collinear emissions relative to the initial parton direction. This scheme is essentially taken over from $e^{+} e^{-}$annihilation and is used in the JADE algorithm for jet reconstruction.

Here, we introduce the ' $z \hat{s}$ ' scheme, which is a variant of the mixed scheme [16]. In the $z \hat{s}$ scheme the soft and collinear divergences with respect to the incoming parton direction are regulated with a cut in $z_{q}$ such that $z_{q \text {,min }}<z_{q}<1-z_{q \text {,min }}$. In addition there is also a cut in the invariant mass of the hard subsystem, $\hat{s}>\hat{s}_{\min }$, which regulates the soft and collinear divergences for the two produced partons. In this way the divergences with respect to the incoming parton direction and with respect to the hard subsystem are treated separately. The advantages of this scheme will become clear in the following.

The difference between these two cut-off schemes is demonstrated in Figs. 2 and 3 . In the $W$ scheme the cut-off in $z_{q}$ is to a good approximation given by $y_{\text {cut }}<z_{q}<1-y_{\text {cut }}$ whereas the cut-off in $\hat{s}$ is given by $\hat{s}>y_{\text {cut }} W^{2}$. Since $\hat{s}=Q^{2}\left(1 / x_{p}-1\right)$ the cut in $\hat{s}$ can be translated into one in $x_{p}$ instead, $x_{p}<Q^{2} /\left(\hat{s}_{\min }+Q^{2}\right)=x /\left(x+y_{\text {cut }}\right)$. This means that if one wants to use the matrix element for small $\hat{s}$ (i.e. large $x_{p}$ ) one also gets very close to the divergences at $z_{q}=0$ and 1 . In the $z \hat{s}$ scheme on the other hand these divergency regions can be avoided and larger $x_{p}$ can be reached since the two divergences are handled separately. This has the advantage that a region with a large $2+1$-jet event rate can be treated with the matrix element. With smaller $\hat{s}$ one can, e.g., reach smaller $x$ in an extraction of the gluon density $g\left(x, Q^{2}\right)$ from the boson-gluon fusion process [18, 19].

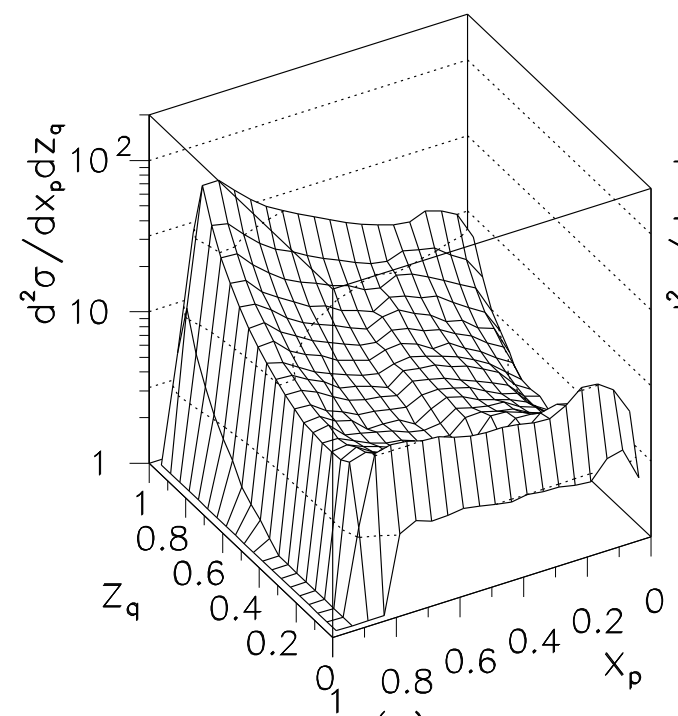

(a)

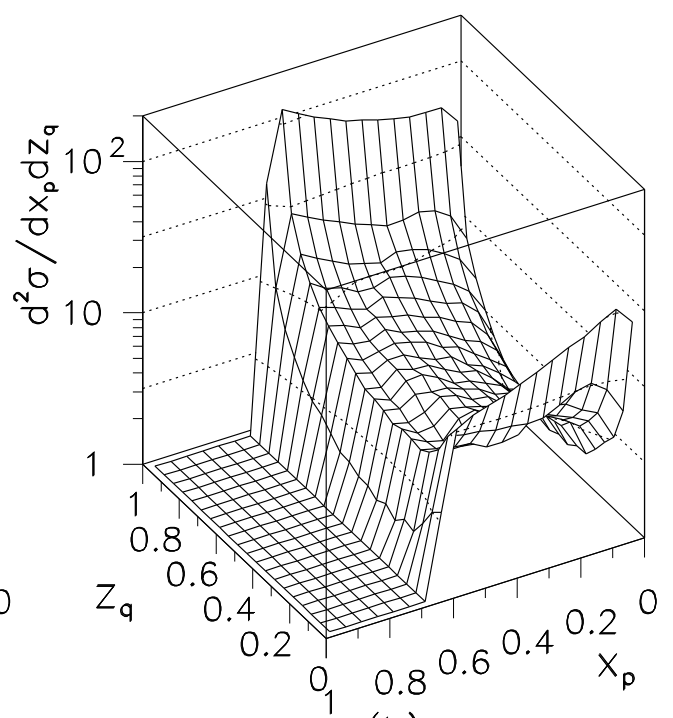

(b)

Figure 2: Differential first order QCD cross-section, eq. (臬), for $x=10^{-3}$ and $Q^{2}=10$ $\mathrm{GeV}^{2}$ in DIS at HERA using (a) the $z \hat{s}$ scheme with $z_{\text {min }}=0.037$ and $\hat{s}_{\text {min }}=1 \mathrm{GeV}^{2}$, (b) the $W$ scheme with $y_{\text {cut }}=0.00062$. The cuts have been chosen very small, such that the first order cross-section equals the total cross-section, to illustrate the behaviour of the cross-section close to the divergences.

Fig. 2 illustrates the $2+1$ parton phase space for the two different schemes with the cut-offs chosen such that the $2+1$ parton cross section equals the total cross-section. From the figure it is obvious that the $W$ scheme has the disadvantage to get very close to the divergences at $z_{q}=0$ and 1 , while the $z \hat{s}$ scheme avoids these regions and instead reaches larger $x_{p}$. 


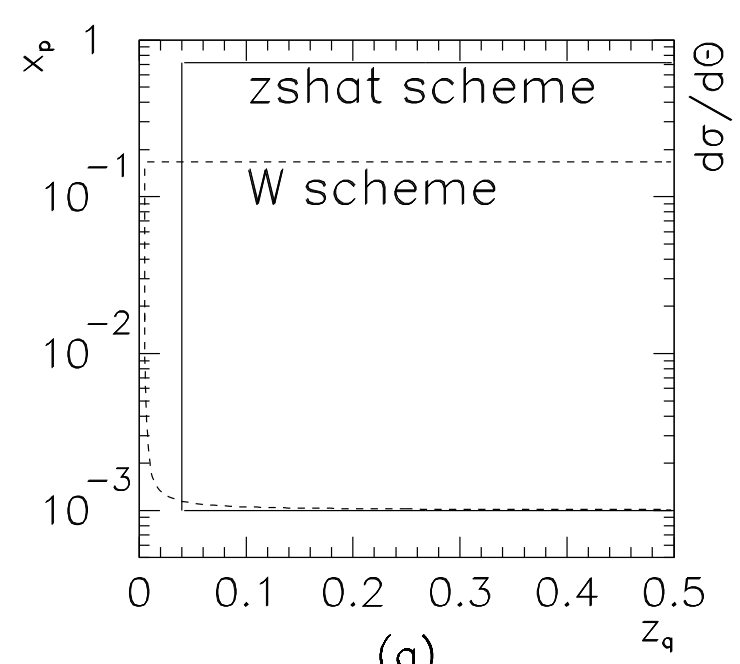

(a)

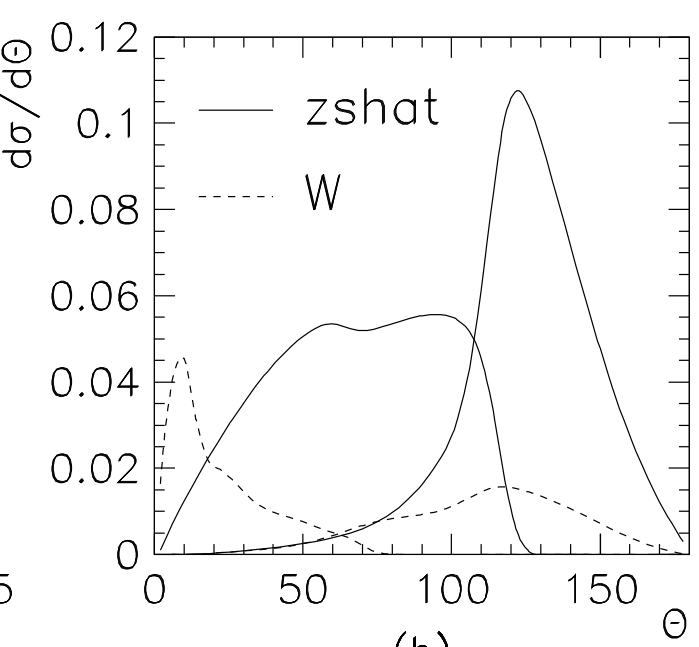

(b)

Figure 3: The 2+1 parton cross-section for $x=10^{-3}$ and $Q^{2}=10 \mathrm{GeV}^{2}$ at HERA using the $z \hat{s}$ scheme (solid) with $z_{\text {min }}=0.04$ and $\hat{s}_{\text {min }}=4 \mathrm{GeV}^{2}$ and the $W$ scheme (dashed) with $y_{\text {cut }}=0.005$. (a) The $\left(z_{q}, x_{p}\right)$ phase space in the two schemes. Note that only the region $z_{q}<0.5$ has been shown due to the symmetry $z_{q} \leftrightarrow 1-z_{q}$ of the cut-offs. (b) Polar angle (with respect to the incoming proton) distribution for the most forward going parton of $j_{1}$ and $j_{2}$ and the other one in the two schemes.

For the following studies we use the cut-offs $z_{\min }=0.04$ and $\hat{s}_{\min }=4 \mathrm{GeV}^{2}$ in the $z \hat{s}$ scheme and $y_{\text {cut }}=0.005$ in the $W$ scheme, resulting in the phase-space region shown in Fig. 3a. Once again this illustrates that one gets much closer to the $z_{q}$ divergences in the $W$ scheme. The same cut-offs has also been used to calculate the angular distributions of the two partons from the matrix elements as shown in Fig. $3 \mathrm{~b}$ which demonstrates a clear difference between the two schemes. In the $W$ scheme there is mostly one very forward parton (along the proton momentum direction) and one backward as opposed to the $z \hat{s}$ scheme which has much fewer forward partons. The reach of smaller $\hat{s}$, in the latter scheme, means that the partons are closer in phase space and thereby in angle.

The fewer forward partons in the $z \hat{s}$ scheme, also imply that forward parton emission should be described by the initial state parton shower, as described in next section. On the other hand, the $W$ scheme has larger $\hat{s}$ and should have more emissions in the final state parton shower.

Since we use lower cuts for the matrix element than in normal jet analyses, where the jet cross-section is usually numerically a fraction $\alpha_{s}$ of the total cross-section, we have to ensure that the jet cross-section is not unphysically large. Large relative jet cross-sections also increase the risk of double counting which for example would affect the energy flow. However, we have explicitly checked that increasing the $\hat{s}$ cut from $4 \mathrm{GeV}^{2}$ to $20 \mathrm{GeV}^{2}$, which makes the jet cross-section be $\sim 20 \%$ of the total, does not influence the energy flows. Still, we want to use the smaller $\hat{s}$ cut to improve the description of small mass diffractive systems with the matrix element.

In the regions of small and large $z_{q}$ where the first order matrix elements diverge rapidly one can expect large corrections from higher orders [20]. Therefore, parton emission in this region should be better treated with parton showers since they include the leading contributions from all orders (see next section). The fact that the $z \hat{s}$ scheme is rather 
flat in the central region of Fig. 2 corresponding to the cut-offs used, shows that one is not getting too close to the divergences. The main principle should be to use the most appropriate approximation, finite order matrix element or leading log parton shower, in each region of the available phase space.

Thus, we use the first order matrix elements with the above defined $z \hat{s}$ cut-off scheme to describe the hard emissions and add parton showers to take into account higher order emissions. The procedure to generate the perturbative interactions in an event is then as follows. First, the overall DIS kinematical variables $x$ and $Q^{2}$ are chosen from the electroweak cross-sections with QCD-improved structure function 13 . Then, the QCD matrix elements are used to choose whether to generate a QCD-Compton or boson-gluon fusion process, or simply a quark-parton model (QPM) quark scattering without an extra parton emission from the matrix elements, i.e. a $q g-, q^{-}$- or $q$-event, respectively. For $q g$ and $q \bar{q}$-events, the matrix element is also used to choose the variables $x_{p}, z_{q}, \phi$ such that the parton four-momenta are fully specified [13]. Parton showers are then added, as will now be discussed.

\subsection{Parton showers}

Higher order parton emissions become very difficult to calculate using exact matrix elements due to the rapidly increasing number of diagrams. Second order matrix elements have been calculated [21], but not implemented in any complete Monte Carlo program for event generation due to problems with a probabilistic interpretation. Higher orders are instead taken into account through parton showers based on the GLAP evolution equations [22] in the leading $\log Q^{2}$ approximation of perturbative QCD. Multiparton emission is thereby factorized into a series of emissions, each described by the basic branching processes $q \rightarrow q g, g \rightarrow g g$ and $g \rightarrow q \bar{q}$, giving an iterative procedure suitable for Monte Carlo simulation. The leading logarithm approximation implies that soft and collinear emissions should be well treated, whereas hard emissions at large angles are not and should instead be treated with matrix elements as far as possible.

The parton showers is a way to take the off-shellness of the incoming and outgoing partons in the matrix element and the associated parton radiation into account. The incoming parton, having a space-like virtuality due to the radiation of time-like or onshell partons in a quantum fluctuation, can be resolved by the hard scattering where the virtuality of the incoming parton is limited by the hardness of the scattering. The initial parton shower then retraces the quantum fluctuation, which is realized by the scattering, back to the originating parton in the incoming nucleon which has a space-like virtuality below the cut-off $Q_{0}^{2}=1 \mathrm{GeV}^{2}$. The partons produced in the hard scattering and by the initial state parton shower may have time-like virtualities $\left(m^{2}>0\right)$ which is reduced in a final state parton shower by branchings into daughter partons with decreasing off-shell masses and decreasing opening angles. Finally all emitted partons have virtualities below a cut-off $m_{0}^{2}=1 \mathrm{GeV}^{2}$ and are put on-shell. The separate treatment of initial and final state parton shower emissions implies the neglect of interference terms between the two and is not gauge invariant, but is consistent with the leading log approximation.

The final state radiation is analogous to parton radiation in $e^{+} e^{-} \rightarrow q \bar{q}$. The well developed and tested algorithm in JETSET [23] is therefore used for all time-like showers in DIS. The technical details are given in [24], but it should be noted that coherence in soft gluon emission is taken into account through angular ordering (decreasing opening angles in subsequent branches). 


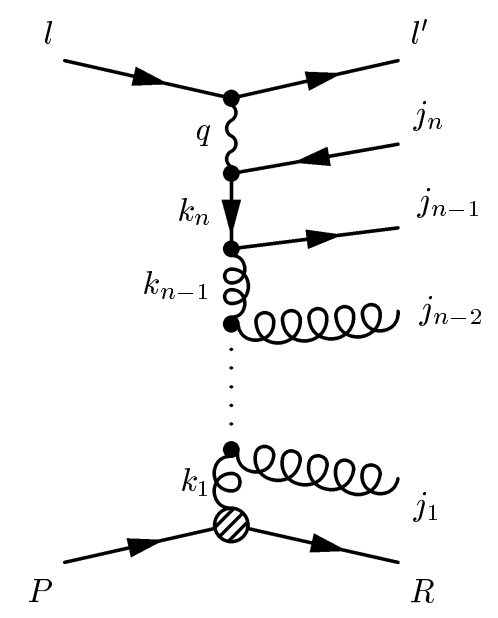

Figure 4: Illustration of a DIS event with higher order parton emission in the parton shower approach. The emitted partons can have time-like virtualities which initiate final state parton showers.

The initial state radiation is performed using the 'backwards' evolution scheme [25]. The shower is then constructed from the hard interaction, associated with the electroweak boson vertex, and evolved backwards with decreasing virtualities down to the essentially on-shell parton from the incoming nucleon. This is a more complicated process, since the nucleon parton density functions must be taken into account in each step. When combining the initial and final state radiation in DIS, special precautions must be taken to preserve energy-momentum conservation and keep the normal definitions of the $x, Q^{2}$ variables [24].

The amount and hardness of the initial and final radiation depends on the off-shellness of the partons entering and emerging from the hard scattering. These virtualities are chosen, using the Sudakov form factor, between the lower cut-offs $\left(Q_{0}^{2}\right.$ and $m_{0}^{2}$, respectively) and a maximum regulated by the hard momentum transfer scale. Having the parton showers as a higher order correction to the matrix element treatment implies adding them on a $q, q g$ or $q \bar{q}$ event. Emissions harder than the ones treated by the matrix elements are then not allowed to avoid double counting. Thus, in the case of a $q$ event the maximum virtuality scale is set by the matrix element cut-off, i.e. $z_{\min } Q^{2} / x$ and $\hat{s}_{\min }$ for the initial and final state parton showers, respectively, in the $z \hat{s}$ scheme or $y_{c u t} W^{2}$ in the $W$-scheme. In the case of a $q g$ or $q \bar{q}$ event the maximum virtuality scale for the final state shower is set to $\hat{s}$. For the initial state shower, the natural scale is the mass-squared of the parton propagator just before the electroweak boson vertex, cf. $\left|k_{n}^{2}\right|$ in Fig. 4 . This is given by the known four-vectors of the electroweak boson and the two final partons from the matrix element. Depending on the underlying Feynman diagram in the amplitude, different combinations are possible leaving some remaining ambiguity and the largest of these possible virtualities has been chosen [13].

Thus the scale for the showers are not simply given by $Q^{2}$, as may be naively expected based on GLAP evolution of structure functions. Instead the relevant scales in the matrix element are used which is quite in analogy with jet physics in photo-production or in hadron-hadron scattering where the $p_{\perp}^{2}$ scale of the jets is used. This means that, at small $x$ in particular, the scale can be significantly larger than $Q^{2}$.

The virtuality scale for the parton showers will vary from event to event, since they 
are regulated by the actual emission in the matrix element treatment. Or, expressed in another way, there is a dynamically changing phase space limit between the matrix element and the parton showers. In each event, all radiation softer than the matrix element emission is given by the parton showers. For $q g$ - and $q \bar{q}$-events this means smaller $z_{q}$ and larger $x_{p}$ in Fig. $\beta$ a and for $q$-events the emissions are outside the matrix-element region in the same figure. The typically smaller $\hat{s}$ in the $z \hat{s}$ scheme, leads to typically less phase space for the final state shower, but instead leave more phase space for the initial state shower. Ideally, the model should be stable against variations of this boundary between matrix element and parton shower treatments. Since these are different approximations in perturbative QCD there will always be some residual dependence on where the boundary between them is drawn, however with the cut-off scheme presently used the model is quite stable when the cut-offs are varied. As emphasized before, the main principle should be to use the most appropriate approximation in each region of the available phase space.

\subsection{Parton level results}

Studies of emitted particles can preferentially be made in the hadronic cms. With the $\log$ arithmic variables rapidity and $\log p_{\perp}^{2}$, the phase space for parton emission has a triangular shape as indicated in Fig. 5. The density of partons from our Monte Carlo model is here shown and compared to the corresponding results of an alternative parton shower approach, the colour dipole model (CDM) [26] as implemented in the ARIADnE Monte Carlo program [7]. The electroweak part is here the same, whereas the QCD parton emission description differs (as well as some aspects of the non-perturbative proton remnant treatment).

In the CDM model, the partons from the hard scattering together with quarks and diquarks in the proton remnant form colour dipoles which radiate additional partons, such that new dipoles are formed and the radiation procedure is iterated. Formally, there is no initial state radiation, but the dipoles radiate in the phase space region normally associated with initial state radiation and therefore effectively simulates both initial and final state radiation. Parton densities are not taken into account, as in the normal GLAP radiation, and there is therefore no related suppression of the 'initial state' radiation. Instead, the CDM model has a suppression due to the extended nature of the proton remnant.

Another important difference between the GLAP-based parton emission scheme and the CDM one, concerns the $p_{\perp}$ ordering of the emitted partons. The GLAP formalism is based on a strong ordering in virtuality of the parton propagators ( $k_{i}$ in Fig. (1) and thereby of the transverse momenta of emitted partons $\left(j_{i}\right)$. This requirement is not present in the CDM model, where the transverse momenta may fluctuate as one is tracing the emissions in rapidity.

Comparing the GLAP-based and CDM-based Monte Carlo results in Fig. Fab, one can clearly see the expected difference. The radiation in the LEPTO model is more suppressed the closer to the proton remnant one gets, whereas the ARIADNE model gives a population of partons which extends more towards the proton remnant. In fact there is only little suppression close to the proton remnant in ARIADNE, such that the radiation is almost symmetric between the proton remnant and the scattered quark. The suppression in LEPTO is partly due to the virtuality ordering in GLAP and partly to the effect of taking the proton's parton densities into account in the initial state evolution. This suppression 

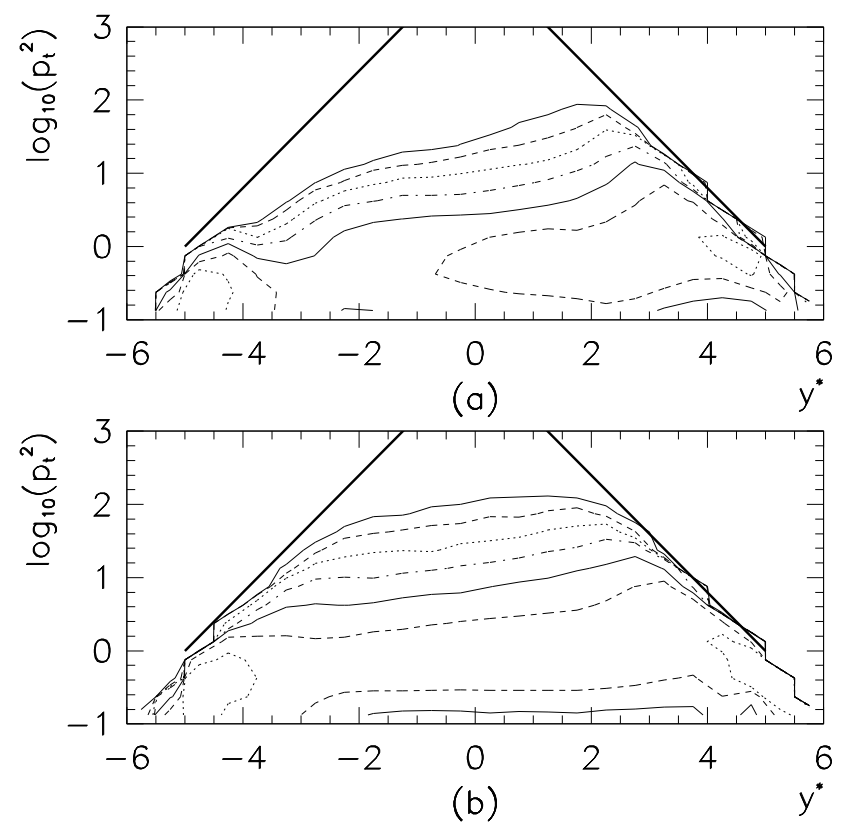

Figure 5: Density $d N / d y^{*} d \log _{10}\left(p_{\perp}^{2}\right)$ of emitted partons in terms of their rapidity $y^{*}$, with the proton remnant at $y^{*}<0$, and transverse momentum squared $p_{\perp}^{2}$ in the hadronic cms as obtained from Monte Carlo events from (a) LEPTO and (b) ARIADNE for $x=10^{-3}$ and $Q^{2}=10 \mathrm{GeV}^{2}$ at the HERA energy. The curves represent lines of constant density of emitted partons with a factor two change between adjacent curves and the same scale in both figures. The thick outer lines indicate the triangular phase space boundary.

is an essential feature of the radiation since the soft colour interactions introduced later cannot give rapidity gaps unless there are gaps already at parton level. The comment in [27] that soft parton radiation should destroy possible gaps depends on the assumption that there is hardly no suppression of the radiation in the dipole model. Taking the parton densities into account the probability will increase for a gap at the parton level.

\section{Non perturbative effects}

To make the transition from the perturbative parton level interactions discussed so far, to the final state of observable hadrons various non-perturbative effects have to be taken into account. This can be achieved by different phenomenological hadronization models. Such models are not based on first principles or derived from the QCD Lagrangian and therefore there are uncertainties and variations possible even in models that are well-functioning in describing many experimental observations.

In the following we discuss two aspects of non-perturbative effects of relevance for DIS. First, the treatment of a target proton remnant containing a sea quark in addition to the valence quarks. Secondly, the newly introduced [11] hypothesis of soft colour interactions which may change the colour structure and thereby produce rapidity gap events. 


\subsection{Proton remnant with a sea quark}

The remnant system is the target nucleon 'minus' the parton entering the hard scattering system (initial parton showers and matrix elements). This interacting parton can be either a valence quark, a sea-quark or a gluon. In case a valence quark is removed the remainder is a diquark which is taken as a colour anti-triplet at the end of a string, to which Lund model hadronization is applied. However, if a sea quark is removed the remainder is more complex, with the valence quarks plus the partner of the interacting sea quark to conserve quantum numbers. Here, we will only discuss the physics aspects of this latter case, where an improved treatment has been introduced [11]. For the other cases and technical details we refer to [13].

The main idea in the new sea quark treatment is that the removed quark is assigned to be a valence or sea quark and, in case of a sea quark, its left-over partner in the remnant is given some dynamics. Thus, the interacting quark is taken as a valence or sea quark from the relative sizes of the corresponding parton distributions $q_{v a l}\left(x_{1}, Q_{1}^{2}\right)$ and $q_{\text {sea }}\left(x_{1}, Q_{1}^{2}\right)$ where $x_{1}$ is the known momentum fraction of the quark 'leaving' the proton and $Q_{1}^{2}$ is the relevant scale (typically the cutoff $Q_{0}^{2}$ of the initial state parton shower). In case of a sea quark, the left-over partner is given a longitudinal momentum fraction from the Altarelli-Parisi splitting function $P(g \rightarrow q \bar{q})$ and the transverse momentum follows from the kinematics once the partner mass has been fixed. Essentially the same results are obtained if the longitudinal momentum fraction is chosen from the corresponding sea quark momentum distribution. The former approach is presently used since this allows the mechanism to be simply implemented in the initial state parton shower routine as an additional, but non-perturbative, $g \rightarrow q \bar{q}$ process.

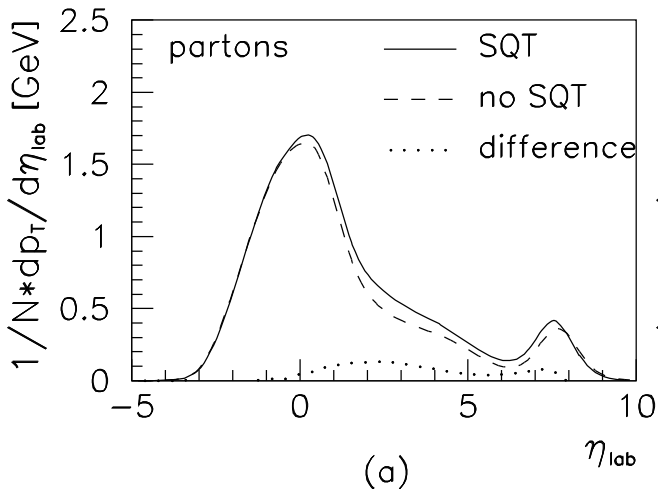

(a)

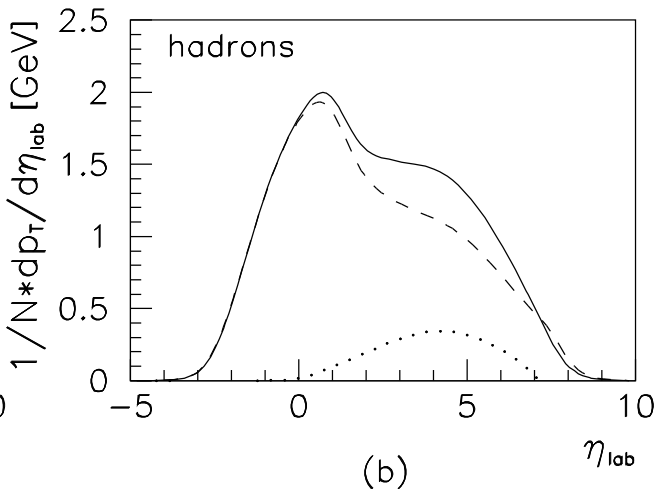

(b)

Figure 6: Transverse energy flow at (a) parton and (b) hadron level from LEPTO with the new target remnant sea quark treatment (SQT, solid curves) and without (dashed curves), as well as their difference (dotted curves); based on simulated HERA events within $0.03<y<0.7$ and $7.0<Q^{2}<70 \mathrm{GeV}^{2}$.

The sea quark partner and the three valence quarks in the remnant, which are split into a quark and a diquark in the conventional way [13], form two colour singlet systems (strings) together with the sea quark partner and the interacting sea quark (and any emitted gluons). This two-string configuration for sea quark initiated processes provides a desirable continuity to two-string gluon-induced events, arising from the boson-gluon fusion matrix element or a $g \rightarrow q \bar{q}$ splitting in the initial state shower. This reduces the 
dependence on the cut-off values in the matrix element and the parton shower. Depending on the partner sea quark momentum, the corresponding string will extend more or less into the central region in rapidity. The hadronization of this extra string will thereby contribute to the forward energy flow, as illustrated in Fig. 6. By comparing the effects of the sea quark treatment on parton and hadron level it is clear that the main effect is due to the extra string between the sea quark partner and the remnant, and not so much from the sea quark partner itself.

A more general conclusion can also be drawn from Fig. 6, namely that the forward energy flow is strongly influenced by hadronization in our model. This is obvious from the large difference between the parton level and hadron level results. It is therefore non-trivial to use this observable as a test of detailed perturbative dynamics, e.g. to discriminate between BFKL and GLAP behaviour.

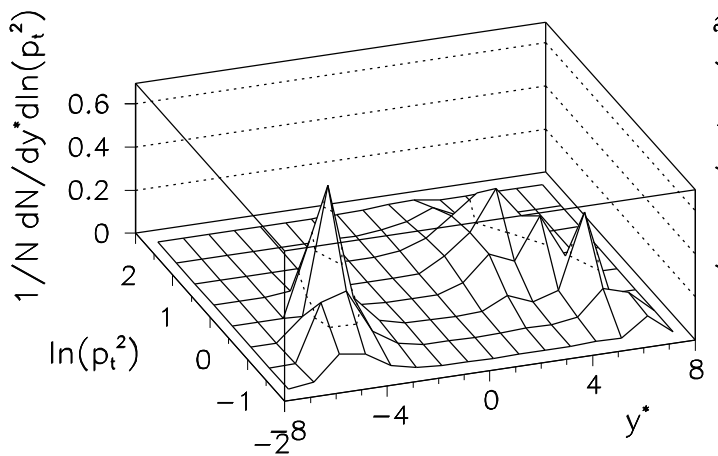

(a)

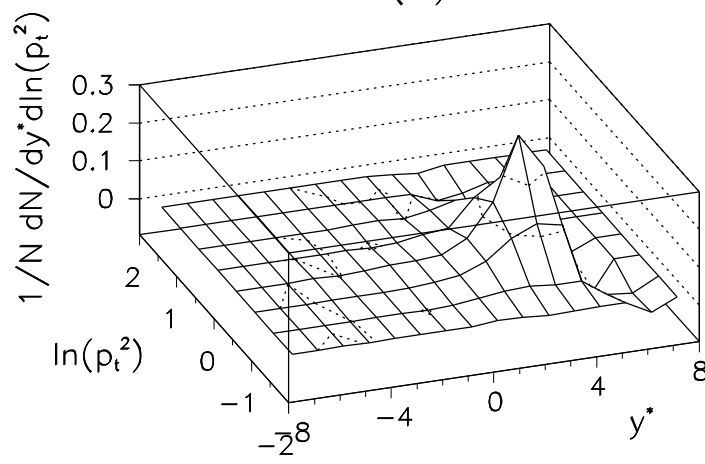

(c)

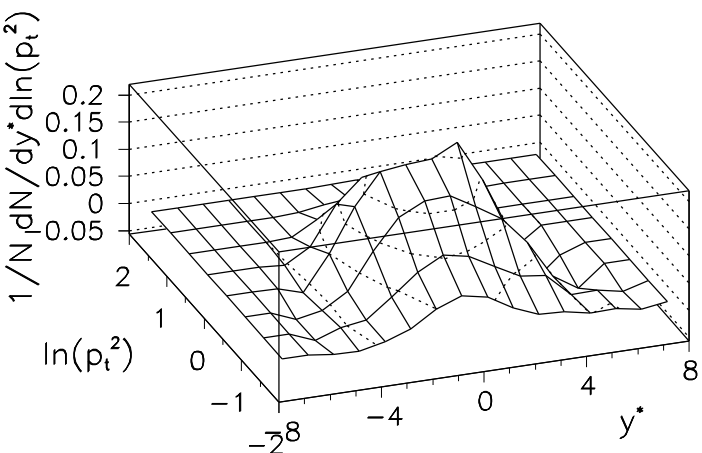

(b)

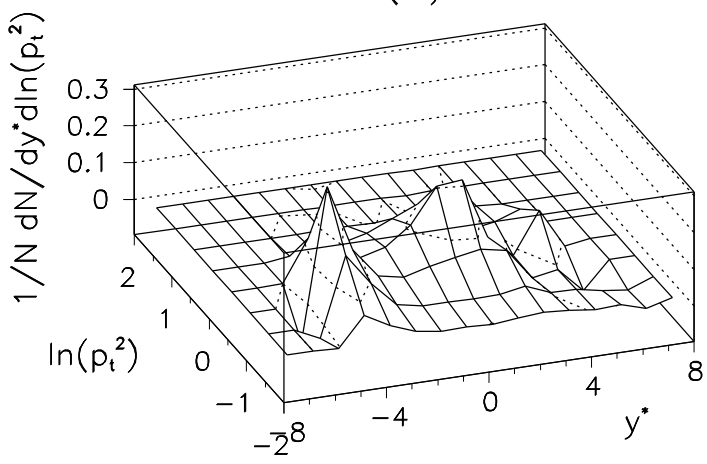

(d)

Figure 7: Distribution in rapidity and transverse momentum (in the hadronic cms) for different emissions in LEPTO: (a) partons from the matrix element (right peak) and in the remnant (left peak), (b) partons from the initial state shower, (c) partons from the final state shower, (d) remnant partons including partner sea quarks. In all cases obtained from simulated HERA events with $x=10^{-3}$ and $Q^{2}=10 \mathrm{GeV}^{2}$.(a) is from a simulation with only the matrix element, while (b)-(d) are the difference of a full simulation and one with the examined effect turned off.

To disentangle the effects of different processes producing partons we turn each of them off and subtract the result from the total simulation and demonstrate the result in Fig. 7 in terms of rapidity and transverse momentum in the hadronic final state. Fig. 7 7 demonstrates that partons from the matrix elements are mainly in the current hemisphere $\left(y^{*}>0\right)$ and have perturbatively large $p_{\perp}$, whereas the remnant partons are 
at large rapidity in the proton direction and have small $p_{\perp}$, as expected. Partons from the initial state shower (Fig. 7 $\mathrm{b}$ ) are distributed as a 'ridge' in rapidity, whereas partons from the final state shower (Fig. $\mathbb{7} \mathrm{c}$ ) are more concentrated in the current region. The remnant partons (Fig. $7 \mathrm{~d}$ ) are mainly at large negative rapidities, but the location of the sea quark partner extends to central rapidities in the hadronic cms. Considering the absolute normalization on the vertical scales, one can note that the main contributions come from the matrix element and the valence partons in the remnant, followed by the initial state parton shower. The relative amount and distribution from matrix element and parton showers does to some extent depend on the cut-off procedure, as discussed before. One should also note that there are negative contributions from the showers and the sea quark treatment. This is just a consequence of conservation of energy. If more particles are produced centrally with non-negligible transverse masses then the 'outer' particles have to be more central to preserve the total hadronic energy, $W$.

\subsection{Soft colour interactions}

The starting point for hadronization is the partonic event generated by the matrix element and the parton showers. This implies a colour separation between the hard scattering system and the proton remnant, which will be connected by colour flux tubes, or strings in the Lund model [28]. As a result of hadronization, the whole rapidity region in between will be populated by hadrons and the probability for a region with no particles is exponentially suppressed with increasing 'gap' size.

The discovery of a rather large fraction $(\sim 10 \%)$ of DIS events at HERA with a large gap in the distribution of final state particles is therefore quite remarkable [4, 5]. These events cannot be understood by conventional DIS models as implemented in LEPTO [6] and Ariadne [7]. They can be reasonably well described by phenomenological models based on pomeron exchange [9, 10], but there is no satisfactory understanding of the pomeron and its interaction mechanism. As an alternative, we have therefore introduced 11] the concept of soft colour interactions (SCI) that give rise to rapidity gap events without using a pomeron from Regge phenomenology.

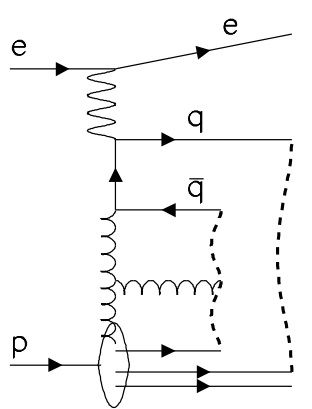

(a)

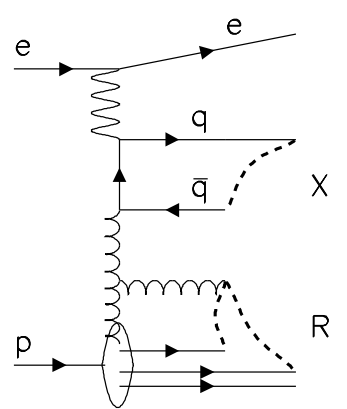

(b)

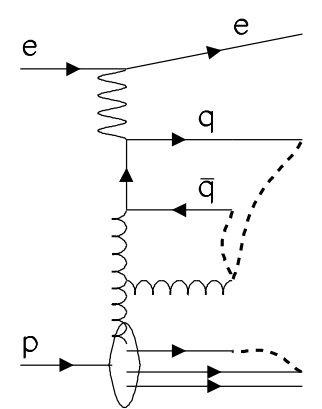

(c)

Figure 8: The string configuration in a gluon-initiated DIS event in (a) conventional Lund string model connection of partons, and (b,c) after reconnection due to soft colour interactions.

The basic idea is here that there may be additional interactions between the partons at a scale below the cut-off $Q_{0}^{2}$ for the perturbative treatment. Obviously, interactions 
will not disappear below this cut-off, the question is how to describe them properly. Our proposed SCI mechanism can be viewed as the perturbatively produced quarks and gluons interacting softly with the colour medium of the proton as they propagate through it. This should be a natural part of the process in which 'bare' perturbative partons are 'dressed' into non-perturbative ones and the formation of the confining colour flux tube in between them. These soft non-perturbative interactions cannot change the momenta of the partons significantly, but they may change their colour and thereby affect the colour structure of the event. This corresponds to a modified topology of the string in the Lund model approach, as illustrated in Fig. 8.

Lacking a proper understanding of non-perturbative QCD processes, we construct a simple model to describe and simulate such soft colour interactions. All partons from the hard interaction plus the remaining quarks in the proton remnant, including a possible sea quark partner, constitute a set of colour charges. Each pair of charges can make a soft interaction changing only the colour and not the momentum, which may be viewed as soft non-perturbative gluon exchange. As the process is non-perturbative the exchange probability for a pair cannot be calculated so instead we describe it by a phenomenological parameter $R$.

The number of soft exchanges will vary event-by-event and change the colour topology of the events such that, in some cases, colour singlet subsystems arise separated in rapidity. In the Lund model this corresponds to a modified string stretching as illustrated in Figs. 8bc, where (b) can be seen as a switch of anticolour between the antiquark and the diquark and (c) as a switch of colour between the two quarks. This kind of colour switches between the perturbatively produced partons and the partons in the proton remnant are of particular importance for the gap formation.

As a result of soft colour interactions, there may be colour singlet systems with so small invariant mass (cf. Fig. 8c) that normal hadronization models are not applicable, since the final state is very constrained and resonance effects are important. This applies in particular to string systems including a valence diquark from the remnant. Such systems are not optimally treated in JETSET regarding the production of one- and two-particle final states and taking isospin constraints into account. For instance, a $\Delta$-resonance is often made instead of a nucleon-pion system. We have therefore constructed a new treatment of such systems in order to account for effective isospin singlet exchange to prevent the production of a single $\Delta$ resonance and instead make two particles if kinematically allowed.

Our procedure is similar to the original one in JETSET, but differs in details which, however, are important for the results. First, all particles in the system are added into a cluster. If the invariant mass of the cluster is large enough then the cluster is decayed into two hadrons isotropically (i.e. according to $d \cos \theta d \phi$ ) in the centre-of-mass system of the cluster. Just as in ordinary string hadronization the flavours in the cluster break are chosen from the $S U(6)$ weights which gives the two hadrons to be formed.

If the cluster mass is too small to make two hadrons, a single hadron is made. The type of hadron to be produced is chosen from the quark and diquark flavours using the $S U(6)$ weights, but applying the mentioned isospin constraint (against $\Delta$-resonances). The invariant mass of the cluster is generally different from the mass of the produced hadron, typically a proton or a neutron, and hence energy and momentum cannot be conserved unless the four-momentum of another parton in the event is also changed. To disturb the parton state as little as possible, the parton $p_{i}$ is chosen which together with the cluster momentum $p_{c}$ gives the largest invariant mass $\left(p_{i}+p_{c}\right)^{2}$. In this way the relative 
changes needed in the four-momenta are very small (typically $\lesssim 10^{-3}$ at HERA). New fourmomenta are then assigned according to $p_{c}^{\prime}=\left(1+\varepsilon_{1}\right) p_{c}-\varepsilon_{2} p_{i}$ and $p_{i}^{\prime}=\left(1+\varepsilon_{2}\right) p_{i}-\varepsilon_{1} p_{c}$, with the conditions that the invariant mass of the other parton is not changed, ${p_{i}^{\prime}}^{2}=p_{i}^{2}$, and that the produced hadron gets the correct mass, $p_{c}^{\prime 2}=m_{h}^{2}$.

In systems of small mass there is a sensitivity on the assumed masses for a quark $\left(m_{q}\right)$ and a diquark $\left(m_{q q}\right)$, as well as the transverse momentum $s_{\perp}$ introduced in the target remnant split [13]. In order not to have a cluster mass larger than the proton mass just from such effects, the quark masses used in JETSET are lowered from 325 to $225 \mathrm{MeV}$. This reduction has been chosen such that $m_{q}+m_{q q}+\sigma_{s} \sim m_{p}$, where the Gaussian width $\sigma_{s}=\sqrt{2\left\langle s_{\perp}^{2}\right\rangle}=0.30 \mathrm{GeV}$ has been chosen from comparison with EMC data on target remnant protons. This, and the resulting properties of the forward-moving $R$-system is discussed in next section.

\section{Observables and data}

\subsection{Remnant characteristics}

The model's treatment of the target remnant can be tested against data on baryon production in the target fragmentation region as obtained in muon-proton scattering by the European Muon Collaboration (EMC). In the analysis of ref. [29], the data on proton and $\Lambda$ production have been analyzed in terms of $\Delta y=\ln \left[(E+|\vec{p}|) / m_{B}\right]$, which is the difference in rapidity between the target proton and the outgoing baryon $B$ and corresponds to having the $z$-axis along the $B$ momentum. The difference of baryons and antibaryons have been taken, to cancel the contribution from baryon-antibaryon pair production in the hadronization and the results are shown in Fig. 9.

The proton spectrum can be well described with both the old and the new models, whereas the $\Lambda$ spectrum is better reproduced with the new model including the improved sea quark treatment and soft colour interactions. In the old sea quark treatment, $\Lambda$ particles were produced directly from the proton remnant and not from a string hadronization which gave a too hard $\Lambda$ spectrum (small $\Delta y$ ). As illustrated, the data also gives some handle on the width $\sigma_{i}$ of the intrinsic transverse momentum associated with the parton's Fermi motion in the target, as well as the Gaussian width $\sigma_{s}$ of the transverse momentum introduced in a target remnant split. This gives further motivation for the chosen values for these parameters, i.e. $\sigma_{i}=0.44 \mathrm{GeV}$ and $\sigma_{s}=0.30 \mathrm{GeV}$.

\subsection{Diffraction and gap events}

The amount of rapidity gaps will depend on the parameter $R$, but as was shown in [11] the dependence is not strong. In fact, increasing $R$ above 0.5 , which we use, does not give an increased gap probability, but may actually decrease it depending on the details of the colour exchanges in the model. This is intuitively understandable, since once a colour exchange with the spectator has occured additional exchanges among the partons need not favour gaps and may even reduce them.

In order to define the $X$ and $R$ systems and compare with experimental data we have used a gap definition similar to [5], i.e. no energy in $\eta_{\max }<\eta<6.6$ with $\eta_{\max }<3.2$. (With $\eta=-\ln \tan \theta / 2$ and $\theta$ the angle relative to the proton beam so that $\eta>0$ is the 


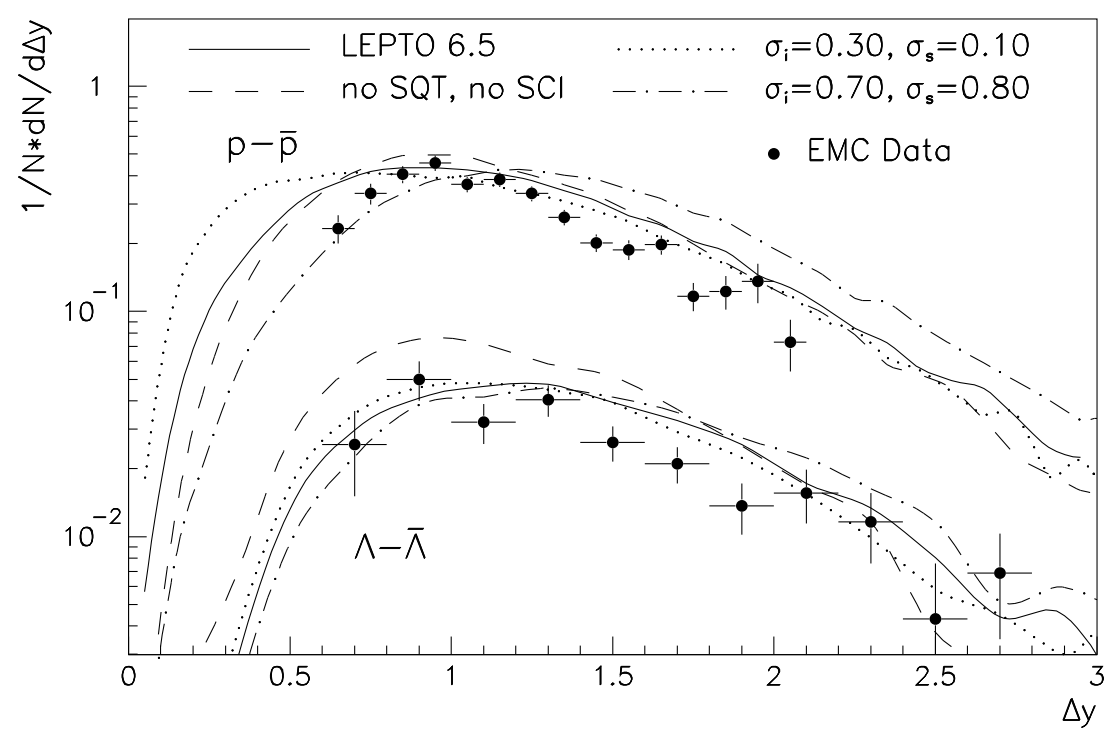

Figure 9: Distribution in rapidity difference, $\Delta y=\ln \left[(E+|\vec{p}|) / m_{B}\right]$, of produced baryons relative to the target proton. The difference baryon-antibaryon enhances the effect of target fragmentation relative to pair production. Data points from EMC and curves from the complete new model with standard parameters (solid) and with varied Gaussian transverse momentum widths $\sigma_{i}$ and $\sigma_{s}$ for intrinsic momentum and target remnant split, as indicated, and the old model without SCI and sea quark treatment (SQT).

proton hemisphere in the HERA lab frame.) The main features of the resulting $R$-system in our model are shown in Fig. 10. Here, $t=\left(p_{p}-p_{R}\right)^{2}$ is the momentum transfer from the incoming proton to the emerging $R$-system, $M_{R}$ is the mass of the remainder system defined as all particles with $\eta>6.6, x_{L}=p_{z} / E_{p}$ is the longitudinal momentum fraction of final state protons.

The $t$-dependence in our model is intimately connected to the assumed distribution of intrinsic transverse momentum $\left(k_{1 \perp}\right.$ in Fig. (1) of partons in the proton, i.e. of the parton entering the hard scattering process. This transverse momentum is balanced by the proton remnant and, since momentum transfers in SCI are neglected, it is essentially the $p_{\perp}$ of the forward $R$-system, i.e. $p_{R, \perp}^{2}=k_{\perp}^{2}$. Now, $t \approx-p_{R, \perp}^{2}$ in the case of interest, i.e. when the energy-momentum transfer from the beam proton to the $X$-system is small giving a very forward $R$-system.

The intrinsic, or primordial, $k_{\perp}$ represents the non-perturbative Fermi motion in the proton and is therefore of the order $k_{\perp} \simeq 1 \mathrm{fm}^{-1}$ or a few hundred $M e V$ as estimated from the uncertainty principle. This gives the width $\sigma_{i}$ of the Gaussian distribution $\exp \left(-k_{\perp}^{2} / \sigma_{i}^{2}\right) d k_{\perp}^{2}$ which is normally assumed. Thus, one directly gets the exponential $t$-dependence $\exp \left(t / \sigma_{i}^{2}\right) d t$ with $\sigma_{i}^{2}=2\left\langle k_{\perp}^{2}\right\rangle$ from the primordial $k_{\perp}^{2}$-distribution. The default value $\sigma_{i}=0.44 \mathrm{GeV}$, obtained from fits to normal DIS data, then gives an effective diffractive-like $e^{5 t}$ distribution as demonstrated in Fig. 10a.

The invariant mass of the forward $R$-system is shown in Fig. 10 b. Here, one should first note that the mass spectrum for the partons in the target remnant peaks around the proton mass, i.e. $\sqrt{\left(p_{q}+p_{d q}\right)^{2}} \sim m_{p}$. The $R$-system is therefore dominantly a single proton, as in a diffractive model based on pomeron exchange, but there is also a tail 


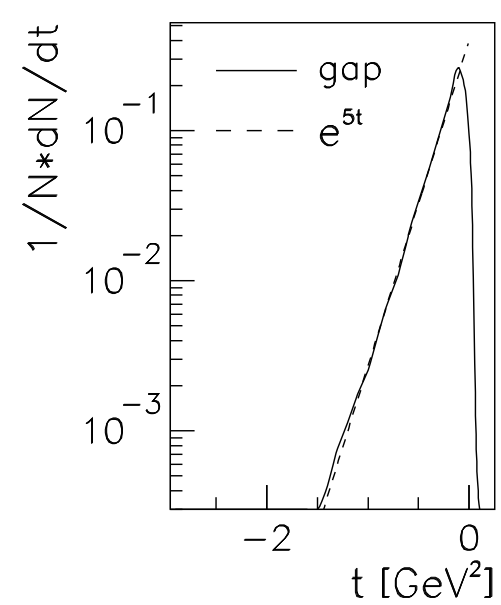

(a)

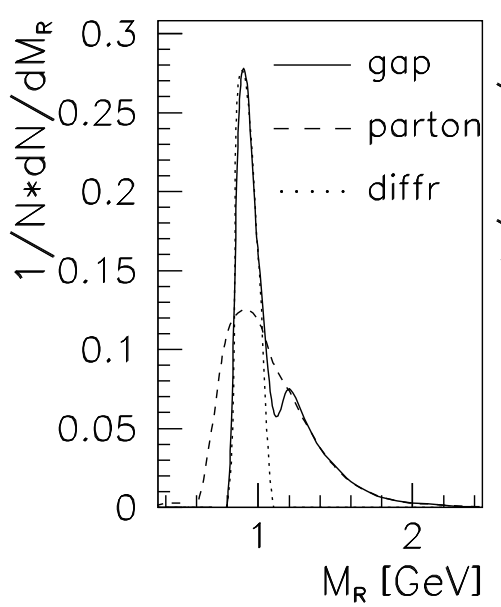

(b)

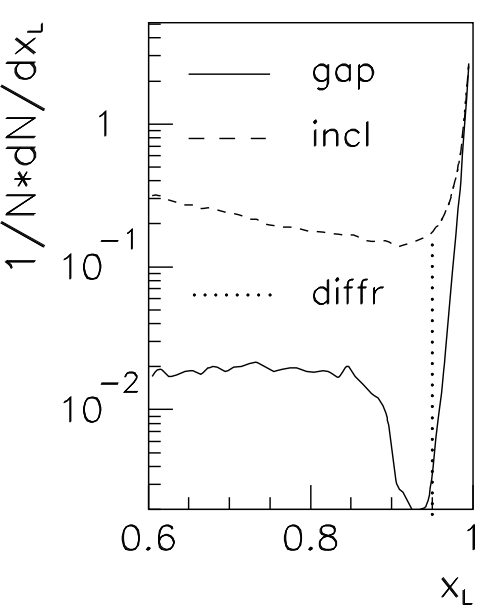

(c)

Figure 10: (a) Squared momentum transfer $t$ from incoming proton to remnant system $R$ for 'gap' events compared with the exponential slope $1 / \sigma_{i}^{2}=5 \mathrm{GeV}^{-2}$. (b) Invariant mass $M_{R}$ of the forward remnant system for 'gap' events at the hadron level (solid line) and parton level (dashed line) compared with 'diffractive' events at hadron level (dotted line). (c) Proton momentum distribution for 'gap' events (solid line), all events (dashed line) and 'diffractive' events (dotted line).

corresponding to the proton remnant dissociating into a larger system. The details of the spectrum depends, however, on the above discussed treatment of small mass systems.

Fig. 10k shows the longitudinal momentum spectrum of protons in our model and demonstrate a clear peak at large $x_{L}$. Based on this distribution we define events having a leading proton with $x_{L}>0.95$ as 'diffractive'. As demonstrated in the figure, most but not all of these events fulfill the above gap definition. In addition to the diffractive peak there are also gap events with protons that have $x_{L}<0.95$. In these events the remnant system typically consists of a nucleon and a pion, cf. the difference of the solid and dotted lines in Fig. 10b. Fig. 10c also shows that there is a clear distinction between the two cases.

Rapidity gaps have been experimentally investigated [4, 50 through the observable $\eta_{\max }$ giving, in each event, the maximum lab pseudo-rapidity where an energy deposition is observed. Fig. 11 shows the distribution of this quantity as obtained from our model simulations for $7.0<Q^{2}<70$ and $0.03<y<0.7$, corresponding to the experimental conditions. In addition, the limit $\eta<3.65$ in the $\mathrm{H} 1$ detector is taken into account. Clearly, the introduction of soft colour interactions have a large effect on the $\eta_{\max }$ distribution. Still, our SCI model is not very sensitive to the exact value of the colour exchange probability parameter $R$ as was shown in [11].

One should note that the basic features of this distribution, the height of the peak and the 'plateau', is in reasonable agreement with the data 田, 50. A direct comparison requires a detailed account of experimental conditions, such as acceptance and varying event vertex position. Selecting events with rapidity gaps similar to the H1 definition (i.e. no energy in $\eta_{\max }<\eta<6.6$ where $\eta_{\max }<3.2$ ) gives the dashed curve in Fig. 11, also in basic agreement with data [5]. The drastic effect of the soft colour interactions on the $\eta_{\max }$ distribution is clearly demonstrated by comparison to the case when they are 


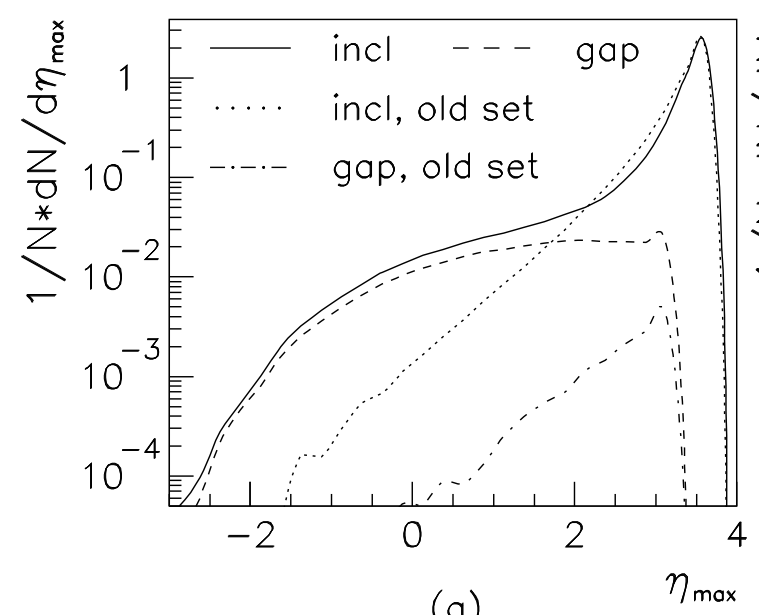

(a)

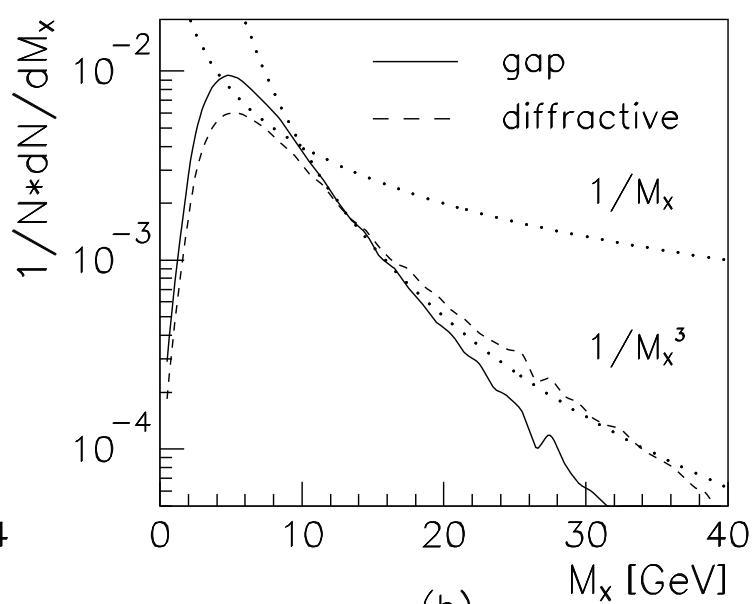

(b)

Figure 11: (a) Distribution of maximum pseudorapidity $\left(\eta_{\max }\right)$. Hadron level after soft colour interactions for all events (solid line) and those satisfying the 'gap' definition (dashed line). The case with no colour reconnections and sea quark treatment is also shown for all events (dotted line) and 'gap' events (dash-dotted line). (b) Invariant mass of the produced central system $X$ for 'gap' events (solid line) and 'diffractive' events (dashed line) compared with a $1 / M_{X^{-}}$and a $1 / M_{X^{-}}^{3}$-distribution.

switched off, which is also included in Fig. 11a.

The $M_{X}$-dependence in Fig. 11]b is close to the $d M_{X} / M_{X}^{3}$ or $d s_{q \bar{q}} / s_{q \bar{q}}^{2}$ behaviour of the BGF matrix element with the cut-off against divergences visible at small $M_{X}$. The large$M_{X}$ region is distorted by the requirement of a gap extending into the central rapidity region. Kinematically, larger $M_{X}$ means a reduced gap and is therefore disfavoured by the gap condition. This can also be seen from the $M_{X}$-distribution for diffractive events where there is no depletion for large $M_{X}$ and instead the distribution follows $d M_{X} / M_{X}^{3}$.

\subsection{The diffractive structure function}

To compare our model with experimental data on rapidity gap events we consider the diffractive structure function $F_{2}^{D}\left(\beta, x_{I P}, Q^{2}\right)$ defined by 30

$$
\frac{d \sigma^{D}}{d \beta d x_{I P} d Q^{2}}=\frac{4 \pi \alpha^{2}}{\beta Q^{4}}\left[1-y+\frac{y^{2}}{2}\right] F_{2}^{D}\left(\beta, x_{I P}, Q^{2}\right)
$$

(i.e. assuming single photon exchange and neglecting $F_{L}$ ). This inclusive quantity contains the dependence on the main variables $\beta \simeq Q^{2} /\left(Q^{2}+M_{X}^{2}\right), x_{I P} \simeq\left(Q^{2}+M_{X}^{2}\right) /\left(Q^{2}+W^{2}\right)$ and the usual DIS momentum transfer squared $Q^{2}$. The acceptance corrected data from H1 [5] are compared in Fig. 12 to our model results obtained by selecting Monte Carlo events with rapidity gaps similar to the $\mathrm{H} 1$ definition (i.e. no energy in $\eta_{\max }<\eta<6.6$ where $\eta_{\max }<3.2$ ).

The model is generally in good agreement with the data. It has a tendency to be below the data at large $Q^{2}$, possibly due to slightly too much parton radiation [11. The $\beta$-dependence seems to be the same in the model and the data and thereby the $M_{X}$ 
dependence is also basically correct. At large $\beta$ there is a slight tendency for the model to be below the data. This is natural since large $\beta$ corresponds to small $M_{X}$ which are not include in the model $\left(\hat{s}_{\min }=4 \mathrm{GeV}^{2}\right)$ since it is outside the perturbative regime.

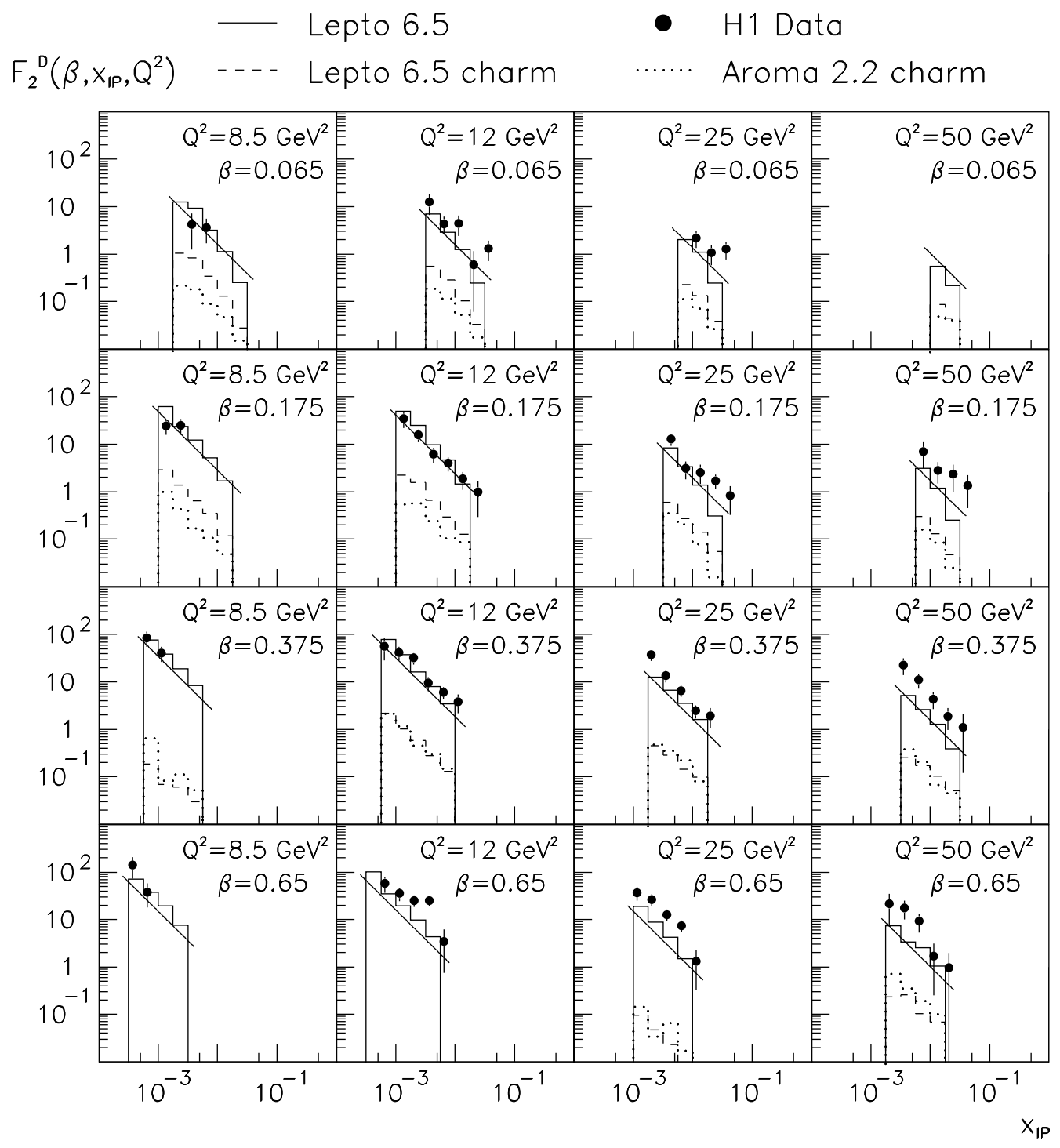

Figure 12: The diffractive structure function $F_{2}^{D}\left(\beta, x_{I P}, Q^{2}\right)$ from our soft colour interaction model using the 'gap' definition (solid histograms) and the 'diffractive' definition (straight lines) compared to the H1 data points [0]. Also shown are the charm contributions in LEPTO (dashed histograms) and AROMA (dotted histograms).

The resulting $x_{\mathbb{P}}$-dependence from the model may be slightly steeper than in the data. Fitting a universal $x_{I P}^{-a}$-dependence we get $a=1.55$ from our model to be compared with the H1 result $a=1.2 \pm 0.1$ [5] and ZEUS results $a=1.3 \pm 0.1$ [4] and $a=1.5 \pm 0.1$ [31] using different methods. However the result obtained in the model depends significantly on which $x_{I P}$-bins that are included in the fitting procedure. This originates from the fact that the $x_{I P}$ dependence in the extracted structure function does not give quite a straight line in this plot, but instead has a tendency for a curvature with smaller slope at small $x_{I P}$ and larger slope at large $x_{I P}$. However, this is not a feature of the model itself but rather a consequence of the selection criteria. Large $x_{I P}$ are suppressed due to the gap 
selection. Changing the limit $\eta_{\max }<3.2$ to $\eta_{\max }<4.2$ the slope changes from $a=1.55$ to $a=1.25$. The latter result is also similar to what is obtained if diffractive events are selected by requiring a leading proton (with $x_{L}>0.95$ ) which gives a slope $a=1.24$. The resulting diffractive structure function with this selection is also indicated with straight lines in Fig. 12. We have also applied the $M_{X}$ method [31] to select diffractive events which gives a slope $a=1.35$. This illustrates that there are large systematic uncertainties when extracting the $x_{I P}$ dependence.

New data covering a larger $\left(\beta, Q^{2}\right)$ range have recently been showed at conferences [32], but the precise numbers are not yet available for direct comparison with our model. The new data seem to indicate that the $x_{I P}$ dependence varies with $\left(\beta, Q^{2}\right)$. In our model the $x_{I P}$ dependence is approximately the same for all $\left(\beta, Q^{2}\right)$ points. The underlying reason for this is that the $x_{I P}$ dependence in the model is basically given by the $\xi$ dependence of the gluon density $\left(F_{2}^{D}\left(x_{I P}\right) \propto x_{I P}^{-1-d} \leftrightarrow \xi g(\xi) \propto \xi^{-d}\right)$ as has also been noted in [12].

An important question is if the selection mechanism together with the acceptance correction can influence the diffractive structure function. The gap selection is dependent on Monte Carlo simulation to extract the efficiency for detecting particles in the very forward region that would destroy the gap. If this efficiency is overestimated then the result would be a too large cross-section. Our model gives more activity in the very forward region than a conventional pomeron based model and thus more gaps are destroyed by particles from the remnant system. As an example of this, the diffractive structure function obtained with our model increases with $\sim 30 \%$ if the forward gap limit is decreased from $\eta=6.6$ to $\eta=5.6$.

There has been some speculation about the charm contribution to the diffractive structure function [33]. As Fig. 12 shows we get a charm contribution of only a few percent in our model. We have also implemented the SCI mechanism in the AromA Monte Carlo [34, which uses the BGF matrix element with explicit charm quark mass included [35]. This gives a factor $\sim 2$ lower charm rate than in LEPTO, where the massless matrix element is used together with a simple threshold factor. However, this difference is not due to the different treatments of the matrix element but rather because in LEPTO the charm sea is also included and gives an additional contribution.

\subsection{Transverse energy flows}

Our model is meant to be applicable for DIS in general and should therefore also be compared with normal DIS events. The observed large forward transverse energy flow in an inclusive event sample requires a substantial energy and particle production and is thereby 'orthogonal' to forward rapidity gaps. In Fig. 13 we compare our model result with the $\mathrm{H} 1$ data [2]. The agreement is quite good except for the smallest $x$-values where the model is below the data in the central region of the hadronic cms.

The soft colour interactions generates not only gap events, but also larger fluctuations in general. In particular, configurations may arise where the string goes 'back and forth' and thereby produces more energy per unit rapidity. The improved treatment of sea quark interactions is also of relevance here, since it gives rise to a string from a valence spectator parton to the partner sea quark remaining in the target system. Depending on the momentum of this sea quark partner, the corresponding string will extend more or less into the central region in rapidity, cf. Figs. 6 and $7 \mathrm{~d}$. The hadronization of this string will thereby give another contribution to the forward energy flow. The effects of 
both these non-perturbative parts of the model are illustrated in Fig. 13 and it is clear that they both give important contributions to an overall better description of the data.

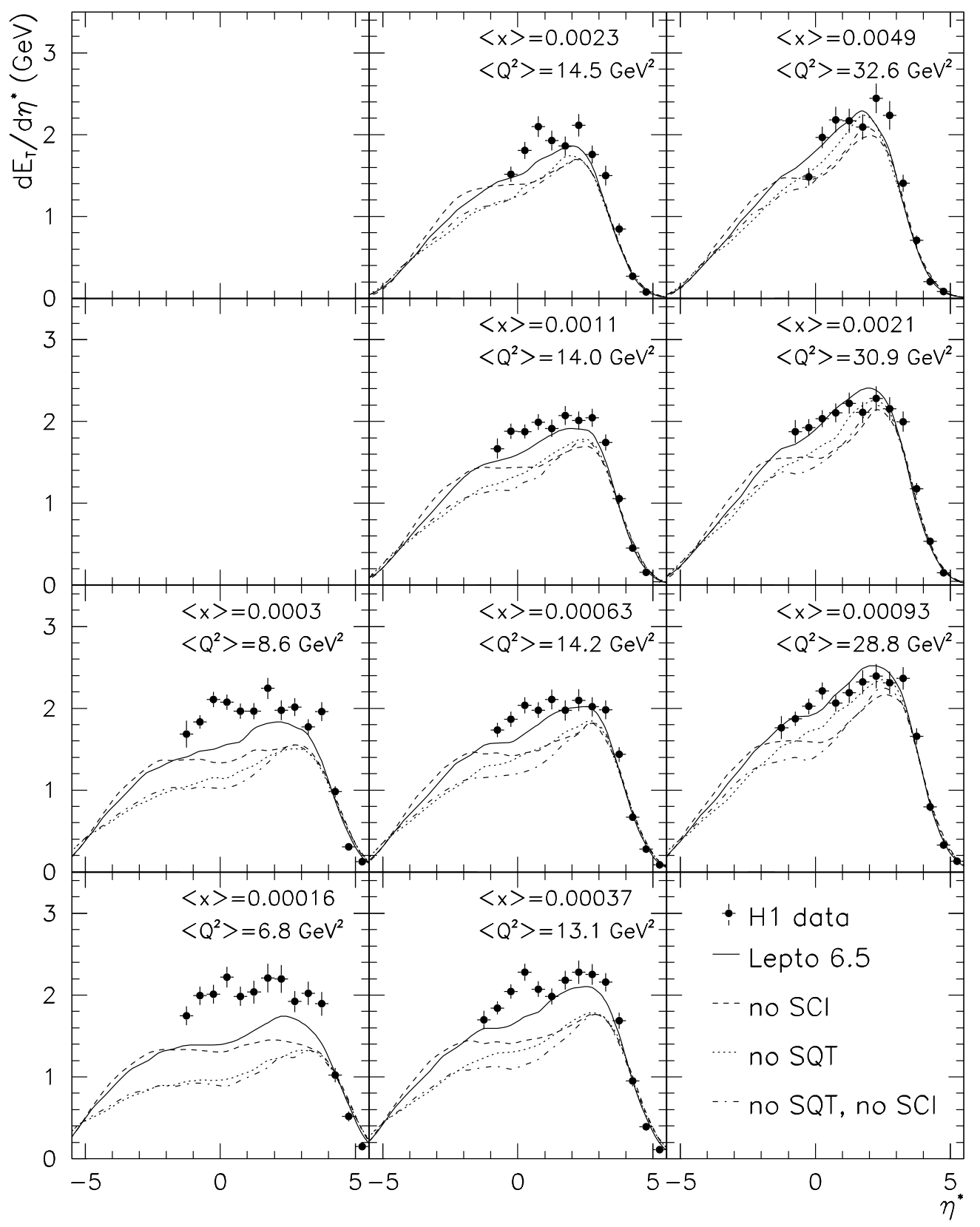

Figure 13: Transverse energy flow versus pseudo-rapidity $\eta^{*}$ in the hadronic cms. H1 data points [2] compared to model curves: the standard LePTo 6.5 model (solid), without soft colour interactions (dashed), without the new sea quark treatment (dotted) and without both of these (dash-dotted).

This also shows the importance of non-perturbative effects for the forward energy flow and makes it less suitable for detailed tests of the underlying perturbative dynamics in terms of, e.g., BFKL or GLAP. This was already demonstrated in Fig. 6 above, in terms of the large difference between the parton and hadron level result.

Finally, we note that the energy flows from the model are not sensitive to the matrix 
element cut-offs in $z_{q}$ and $\hat{s}$, which can be varied substantially without giving a significant effect (not explicitly shown in Fig. 13). This is very satisfactory, since these cut-offs are just a boundary between two different calculational approximations, i.e. the first order matrix elements and the parton showers.

\section{Concluding discussion}

The observed large forward transverse energy flow has been argued [36] to be a sign of BFKL dynamics as predicted in perturbative QCD at small- $x$. We have here demonstrated that this observable is largely influenced by non-perturbative effects. In addition to normal hadronization, there are also significant effects related to the treatment of the proton target remnant when a sea quark interacted, which is more likely at small $x$, as well as the soft colour interactions. This makes is difficult to conclude whether the observed large forward transverse energy flow should be taken as evidence for BFKL behaviour.

In our model we are using the conventional GLAP evolution scheme [22] summing leading $\log Q^{2}$ terms. Terms with $\log (1 / x)$ are neglected in GLAP, but will become important at small enough $x$ and should then be resummed as in the BFKL equation [1]. Therefore GLAP evolution should no longer be valid at some small $x$. Recent studies [37] based on the CCFM equation [38] which sums both leading $\log Q^{2}$ and $\log (1 / x)$ terms suggests that this happens only at very small $x$, below the region accessible at HERA. Thus, there is no strong reason that the GLAP formalism should not be applicable for our purposes. Indeed, with the inclusion of the non-perturbative effects, our model is also able to reproduce the observed forward energy flow reasonably well.

Concerning the interpretation of the observed rapidity gap events, a major issue is whether only pomeron-based models are capable of describing the data or other models can do it as well. Our model [1] which is elaborated upon in this paper, is based on conventional DIS with perturbative QCD matrix elements and parton showers, but with the additional assumption of soft colour interactions before the normal Lund hadronization model is invoked.

It should here be noted that other types of soft interactions have been discussed in other contexts. Colour reconnections causing modified Lund string topology has been investigated [39] in case of $e^{+} e^{-} \rightarrow W^{+} W^{-} \rightarrow q_{1} \bar{q}_{2} q_{3} \bar{q}_{4}$, where the two resulting strings may interact. Soft interactions of a colour charge moving through a colour medium has been considered in [40], and argued to give rise to large K-factors in Drell-Yan processes and synchrotron radiation from the QCD vacuum. Although these studies are not related to rapidity gaps, they contribute to a more general attempt to understand non-perturbative QCD.

Returning to the rapidity gap problem, one may worry [27] that unsuppressed parton emission will populate the rapidity region such as to destroy the gaps, or lower their probability below the observed one. The cut-off in parton virtuality, defining the borderline to the non-perturbative region, is a regulator of this. As discussed in section 2, our model has the standard values for such cut-offs and there is no need for an extra suppression of the perturbative emission. Nevertheless, we obtain a significant rate of gap events, in basic agreement with data.

Of importance for the gap rate is also the fluctuations in the initial parton emission [11]. Although, one may expect that a GLAP parton shower gives a fair mean description of 
events, there is no guarantee that it accounts properly for fluctuations. Larger fluctuations of the number of emitted gluons would increase the rate of gap events and also increase the inclusive forward energy flow due to 'downwards' and 'upwards' fluctuations, respectively.

If this new attempt to understand the rapidity gap phenomenon is successful in further detailed comparison with data, it will circumvent some problems in the pomeron-based approach. In particular those associated with the concept of a preformed exchanged object. This object cannot be a real particle or state, since it has a negative mass square $t$. It could be a virtual exchange corresponding to some real state, such as a glueball, but this is presently unclear (although a recent glueball candidate [41] fits on the pomeron trajectory). The interpretation of factorization in Regge phenomenology in terms of an emission of a pomeron given by a pomeron flux and a pomeron-particle interaction crosssection also has some problems. Since it is only their product that is experimentally observable one cannot, without further assumptions, define the absolute normalization of this flux and cross-section unambiguously 42. This also gives a normalization ambiguity for the parton densities of the pomeron which is reflected in the problem of whether they fulfill a momentum sum rule or not.

Leaving the concept of a preformed object and instead considering [43] a process with interactions with the proton both before and after the hard scattering (taking place at a short space-time scale) may avoid these problems, as in our model. One may ask whether the soft colour interactions introduced here is essentially a model for the pomeron. This should not be the case as long as no pomeron or Regge dynamics is introduced in the model. At present, the model has only one new free parameter, the colour exchange probability $R$. Other parameters belong to the conventional DIS model [13] and have their usual values.

Our model attempts to describe both diffractive and inclusive interactions in a unified framework. Thus, data on both aspects should be used to test it. Typical diffractive characteristics concern properties of the forward $R$-system and the $M_{X}$-distribution. Here, one should investigate the dependence on the size of the rapidity gap and leading particle

requirements. Pomeron-based models, which are constructed with a leading proton and a gap, are likely to be less sensitive compared to our model, where the gap size depends on fluctuations in parton emissions and soft interactions and the $R$-system is less constrained.

In conclusion, we have shown that it is possible to describe two very different observations, namely large forward energy flows in DIS and a substantial fraction of events with a large rapidity gap, within one unified framework based on standard perturbative QCD and hadronization plus the novel assumption of soft colour interactions.

\section{References}

[1] E.A. Kuraev, L.N. Lipatov, V.S. Fadin Sov. Phys. JETP 45 (1977) 199

Ya. Balitsky, L.N. Lipatov, Sov. J. Nucl. Phys. 28 (1978) 822

[2] T. Ahmed et al., H1 Collaboration, Z. Phys. C63 (1994) 377;

Phys. Lett. B356 (1995) 118

[3] M. Derrick et al., ZEUS collaboration, Phys. Lett. B338 (1994) 483

[4] M. Derrick et al., ZEUS collaboration, Phys. Lett. B315 (1993) 481; Z. Phys. C68 (1995) 569 
[5] H1 collaboration, Nucl. Phys. B429 (1994) 477, Phys. Lett. B348 (1995) 681

[6] G. Ingelman, LePto 6.1, in proc. 'Physics at HERA', DESY 1991, p. 1366

[7] L. Lönnblad, Ariadne version 4, Comp. Phys. Comm. 71 (1992) 15

[8] G. Ingelman, P.E. Schlein, Phys. Lett. B152 (1985) 256

[9] P. Bruni, G. Ingelman, Pompyt unpubl. program manual P. Bruni, G. Ingelman, in proc. International Europhysics conf. of High Energy Physics, Marseille 1993, Eds. J. Carr,M. Perrottet, Editions Frontiers, p. 595

[10] H. Jung, Comp. Phys. Commun. 86 (1995) 147

[11] A. Edin, G. Ingelman, J. Rathsman, Phys. Lett. B366 (1996) 371

[12] W. Buchmüller, A. Hebecker, Phys. Lett. B355 (1995) 573

[13] G. Ingelman, A. Edin, J. Rathsman, LePTo 6.5, DESY 96-057, see also WWW page http://www3.tsl.uu.se/thep/lepto/

[14] J. Botts et al., Phys. Rev. D51 (1995) 4763

[15] A. Méndez, Nucl. Phys. B145 (1978) 199

R. Peccei, R. Rückl, Nucl. Phys. B162 (1980) 125

Ch. Rumpf, G. Kramer, J. Willrodt, Z. Phys. C7 (1981) 337

[16] J.G. Körner, E. Mirkes, G.A. Schuler, Int. J. Mod. Phys. A4 (1989) 1781

[17] M. Seymour, Comp. Phys. Commun. 90 (1995) 95

[18] A. Edin, TSL/ISV-93-0087

[19] S. Aid et al., Nucl. Phys. B449 (1995) 3

[20] E. Mirkes, D. Zeppenfeld, TTP95-42, MADPH-95-916, hep-ph/9511448

E. Mirkes, D. Zeppenfeld, TTP96-10, MADPH-96-935, hep-ph/9604281

[21] T. Brodkorb, J. G. Körner, Z. Phys. C54 (1992) 519, T. Brodkorb, J. G. Körner and E. Mirkes, Nucl. Phys. B29A (1992) 144, T. Brodkorb and E. Mirkes, Z. Phys. C66 (1995) 141

D. Graudenz, Phys. Lett. B256 (1991) 518; Phys. Rev. D49 (1994) 3291

[22] V.N. Gribov, I.N. Lipatov, Sov. J. Nucl. Phys. 15 (1972) 438

G. Altarelli, G. Parisi, Nucl. Phys. B126 (1977) 298

[23] T. Sjöstrand, Comp. Phys. Comm. 82 (1994) 74

[24] M. Bengtsson, T. Sjöstrand, Z. Phys. C37 (1988) 465

M. Bengtsson, G. Ingelman, T. Sjöstrand, in proc. HERA Workshop 1987, Ed. R.D. Peccei, DESY Hamburg 1988, Vol. 1, p. 149

[25] T. Sjöstrand, Phys. Lett. 157B (1985) 321

M. Bengtsson, T. Sjöstrand, M. van Zijl, Z. Phys. C32 (1986) 67

[26] B. Andersson, G. Gustafson, L. Lönnblad, U. Pettersson, Z. Phys. C43 (1989) 621 
[27] L. Lönnblad, hep-ph/9512373, to appear in the proceedings from 'HERA Physics Proton, Photon and Pomeron Structure', Durham, September 1995

[28] B. Andersson et al., Phys. Rep. 97 (1983) 31

[29] T. Dreyer, M. Erdmann (Freiburg U.), FREIBURG-THEP-91-8, July 1991

[30] G. Ingelman, K. Prytz, Z. Phys. C58 (1993) 285

[31] M. Derrick et al., ZEUS collaboration, DESY 96-018

[32] A. Mehtha, H1 collaboration, talk given at conf. Topical Conference on Hard Diffractive Processes, February 1996, Eilat, Israel

[33] T. Gehrmann, W.J. Stirling, Durham preprint DTP-95-26-REV

M. Genovese, N.N. Nikolaev, B.G. Zakharov, DFTT-77-95

[34] G. Ingelman, J. Rathsman, G.A. Schuler, Aroma 2.2, DESY 96-058, see also WWW page http://www3.tsl.uu.se/thep/aroma/

[35] G.A. Schuler, Nucl. Phys. B299 (1988) 21.

[36] K. Golec-Biernat, et al., Phys. Lett. B335 (1994) 220; Phys. Rev. D50 (1994) 217

[37] B. Andersson et al., Lund preprint LU TP 95-34

[38] M. Ciafaloni, Nucl. Phys. B296 (1988) 49

S. Catani, F. Fiorani, G. Marchesini, Phys. Lett. B234 (1990) 339;

Nucl. Phys. B336 (1990) 18

[39] G. Gustafson, U. Pettersson, P. Zerwas, Phys. Lett. B209 (1988) 90

T. Sjöstrand, V. Khoze, Z. Phys. C62 (1994) 281

G. Gustafson, J. Häkkinen, Z. Phys. C64 (1994) 659

[40] O. Nachtmann, A. Reiter, Z. Phys. C24 (1984) 283

G.W. Botz, P. Heberl, O. Nachtmann, Z. Phys. C67 (1995) 143

[41] WA91 Collaboration, Phys. Lett. B324 (1994) 509, ibid. B353 (1995) 589

[42] P.V. Landshoff, Introduction to the Pomeron Structure Function, hep-ph/9505254, in proc. Workshop on DIS and QCD, Paris 1995, Eds. J.F. Laporte, Y. Sirois, Ecole Polytechnique, p. 371

[43] A. Berera, D.E. Soper, Phys. Rev. D50 (1994) 4328 